PLANETARY SCIENCE

\section{Facelift for Triton}

Icarus http://doi.org/rgc (2014)

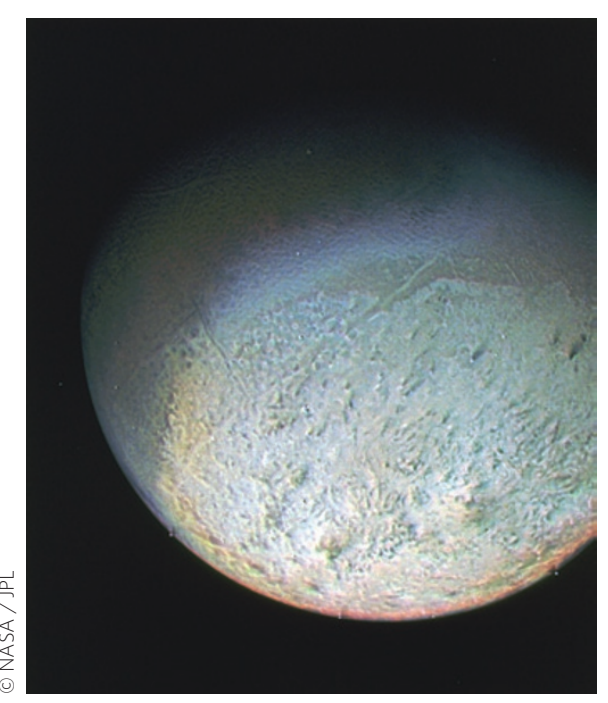

Triton, Neptune's largest moon, is thought to have formed in the Kuiper Belt, the icy region of the Solar System that lies beyond the planets. Numerical modelling of the thermal evolution of Triton suggests that its geologically active surface could be shaped by tidal heating because of its unusual orbit around Neptune.

Francis Nimmo at the University of California, Santa Cruz, USA, and John Spencer at the Southwest Research Institute, USA, assessed the interior evolution of Triton following its capture by Neptune. Assuming that Triton's youthful surface with few craters - as imaged by the Voyager 2 spacecraft - requires convection, a present-day source of heating is required to permit a long-lived ocean beneath a convecting ice shell. The researchers use analytical and numerical models to show that heating generated by tidal sloshing within the ocean is sufficient to drive deformation of the icy surface when Triton's unusually oblique orbit is taken into account.

Although Pluto also formed in the Kuiper Belt, the researchers predict that similar tidal heating is unlikely to occur on the dwarf planet, suggesting that NASA's New Horizon mission - expected to arrive at Pluto in 2015 - may encounter few signs of recent geological activity.

TG

\section{CORE PROCESSES}

\section{Patchy polarity}

Phys. Earth Planet. Inter. http://doi.org/rvp (2014)

Over Earth's history, the geomagnetic field has intermittently reversed direction, so that the positions of the magnetic north and magnetic south poles swap. Numerical simulations of Earth's dynamo - the driver of the magnetic field - suggest that the frequency of these magnetic reversals is linked to convection in the lower mantle.

Peter Olson at Johns Hopkins University, USA, and Hagay Amit at the University of Nantes, France, use calculations to assess how the turbulent movements in Earth's liquid outer core that power the dynamo are affected by the flux of heat across the boundary between the core and mantle. They use seismic images of the present-day lower mantle, which identify patches of warmer and cooler mantle, to constrain the pattern of heat flux across the core-mantle boundary. The researchers find that when the transfer of heat across the boundary is high and heterogeneous, convection in the
BIOGEOCHEMISTRY

\section{Yellow River erosion}

Biogeosciences 11, 945-959 (2014)

Soil erosion can result in the redistribution of substantial quantities of organic matter within a watershed. A study of sediment dynamics in the Yellow River basin, China, suggests that much of the soil organic carbon dislodged by erosion is buried within the river itself.

$X_{i} X_{i}$ Lu of the National University of Singapore and colleagues assessed the effect of soil erosion on sediment and organic carbon fluxes in the Yellow River basin between 1950 and 2010. Using records of soil erosion, together with data on sediment loading and soil and sediment organic carbon content, they estimate that over $130 \mathrm{Gt}$ of soil, which comprised over $1 \mathrm{Gt}$ of organic carbon, was eroded over this period. Of the organic carbon released, around $50 \%$ was buried in the river system, much of it behind dams, and around $23 \%$ was delivered to the Bohai Sea. The researchers suggest that the rest was broken down during erosion and transport processes, and subsequently released to the atmosphere.

Less carbon would have been captured within the river basin, and more delivered to the ocean or lost to the atmosphere, were it not for the construction of dams and the implementation of soil control measures, such as the conversion of hillslopes into terraces. AA

outer core becomes very turbulent, causing the magnetic field to destabilize and reverse more frequently.

Because the heat flux at the core-mantle boundary is regulated by convection in the mantle, reversals of Earth's magnetic field must also be linked to changes in the vigour and pattern of mantle convection.

\section{CRYOSPHERE}

\section{Bobbing for sea ice}

Geophys. Res. Lett. http://doi.org/rvn (2014)

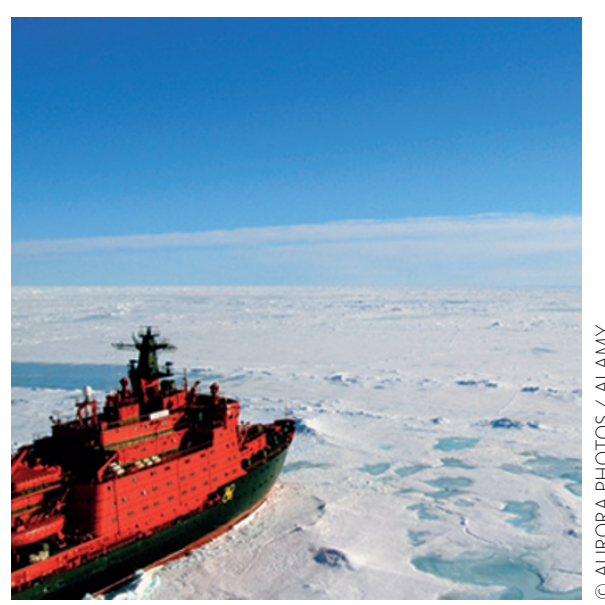

The extent of Arctic sea-ice cover during the summer melt season has declined sharply over the past decade or two. Iceloss measurements show that the sea ice near the North Pole has, at least so far, not experienced substantial losses.

Don Perovich at the US Army Cold Regions Research and Engineering Laboratory and colleagues examined annual losses in sea ice between the North Pole and Greenland as a result of summer melt over the past 14 years, as measured by autonomous buoys tracking sea-ice mass balance. They found large variability in melt between years at both the bottom and top of the sea ice. Also, the ice remained over a metre thick at the end of each melt season. They suggest that the survival of sea ice in the region can be attributed to the lower solar radiation reaching the surface at these high latitudes, compared to the periphery of the sea ice cap.

Although the measurements show no definitive increase in sea-ice melt in the North Pole over the study period, the largest amounts of bottom melt have occurred in the past few years, an observation often associated with rapid sea-ice loss and indicative of a warming ocean.

Written by Anna Armstrong, Tamara Goldin and Amy Whitchurch 